\title{
FRAME-BASED IMAGE DEBLURRING WITH UNKNOWN BOUNDARY CONDITIONS USING THE ALTERNATING DIRECTION METHOD OF MULTIPLIERS
}

\author{
Mariana S.C. Almeida and Mário A. T. Figueiredo \\ Instituto de Telecomunicações and \\ Instituto Superior Técnico, Technical University of Lisbon, Portugal
}

\begin{abstract}
The alternating direction method of multipliers (ADMM) is an efficient optimization tool that achieves state-of-the-art speed in several imaging inverse problems, by splitting the underlying problem into simpler, efficiently solvable sub-problems. In deconvolution, one of these sub-problems requires a matrix inversion, which has been shown to be efficiently computable (via the FFT), if the observation operator is circulant, i.e., under periodic boundary conditions. We extend ADMM-based image deconvolution to a more realistic scenario: unknown boundaries. The observation is modeled as the composition of a periodic convolution with a spatial mask that excludes the regions where the periodic convolution is invalid. We show that the resulting algorithms inherit the convergence guarantees of ADMM and illustrate its performance on non-periodic deblurring under frame-based regularization.
\end{abstract}

Index Terms - Image deconvolution, alternating direction method of multipliers (ADMM), boundary conditions, non-periodic deconvolution, inpainting.

\section{INTRODUCTION}

In image deconvolution, the pixels located near the boundary of the observed image depend on pixels (of the unknown image) located outside of its domain. Despite that, an adequate treatment of these unobserved boundary pixels is almost always avoided in the literature, where the deconvolution is typically tackled by assuming one of the classical boundary condition (BC) [17], such as zero (or Dirichlet), reflexive (or Neumann), or anti-reflexive [10]. For the sake of simplicity and computational convenience, most fast deconvolution algorithms assume periodic BC, which has the advantage of allowing convolutions to be efficiently carried out using the FFT. However, as illustrated in Fig. 1, these BC are quite unnatural and are not accurate models of most real imaging systems. Deconvolution algorithms that ignore this mismatch and wrongly assume periodic BC lead to the well known boundary artifacts. A better assumption about the image boundaries is simply that they are unobserved/unknown, which models well a canonical imaging system where an image sensor captures the cental part of the image projected by the lens.

Deconvolution algorithms based on the alternating direction method of multipliers (ADMM [14, 5]) hold the state-of-the-art in terms of speed, having been shown (see, e.g., [1,2]) to be considerably faster than the classical iterative shrinkage-thresholding (IST) algorithms [9, 12] and their accelerated versions [3, 4, 23]. The

This work was partially supported by Fundação para a Ciência $e$ a Tecnologia (FCT), under grants PTDC/EEA-TEL/104515/2008, PEstOE/EEI/LA0008/2013, PTDC/EEI-PRO/1470/2012 and the fellowship SFRH/BPD/69344/2010.

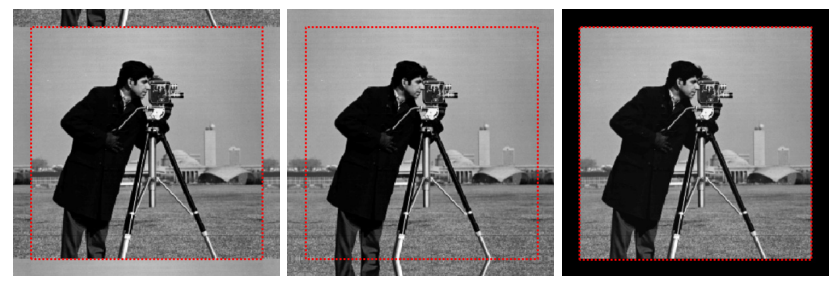

Fig. 1. Illustration of the (unnatural) assumptions underlying the periodic, reflexive, and zero boundary conditions.

standard ADMM-based approach to image deconvolution involves a sub-problem that requires a matrix inversion. This inversion can be efficiently computed in the case of periodic BC (as shown in $[1,2]$ ), but not if we assume unknown boundaries. In this paper, we address this issue by factoring the observation operator into a convolution and a spatial mask that keeps only those pixels that no not depend on the boundary values. We propose an instance of ADMM in which these two operations are decoupled into separate sub-problems that can be efficiently handled.

Assuming unknown boundaries to avoid the boundary artifacts in image deconvolution has been proposed by several authors $[6,16$, $19,22]$. In [19], unknown boundaries were considered in the context of quadratic (Tikhonov) regularization and it was shown that the corresponding matrix inversion can be reduce to an FFT-based inversion followed by the solution (via, e.g., conjugate gradient - CG) of a much smaller system (of the same dimension as the unknown boundary). Very recently, [22] adapted this technique to deconvolution under non-smooth regularization, by proposing an algorithm based on variable splitting and quadratic penalization. That method is related to, but it is not ADMM, thus has no convergence guarantees. Recent work in [16] proposes an approach for deblurring with unknown boundaries, using MFISTA [3], which is known to be slower than ADMM-based methods [1].

\section{ADMM}

Following [13], consider the unconstrained minimization problem

$$
\min _{\mathbf{z} \in \mathbb{R}^{d}} \sum_{j=1}^{J} g_{j}\left(\mathbf{H}^{(j)} \mathbf{z}\right),
$$

where $\mathbf{H}^{(j)} \in \mathbb{R}^{p_{j} \times d}$ are arbitrary matrices, and $g_{j}: \mathbb{R}^{p_{j}} \rightarrow \mathbb{R}$ are convex functions. The instance of ADMM proposed in [13] to solve (1) is presented in Algorithm 1, where $\boldsymbol{\zeta}^{(j)} \in \mathbb{R}^{p_{j}}$ denotes the $j$-th 
block of $\zeta$ in the following partition

$$
\boldsymbol{\zeta}=\left[\begin{array}{c}
\boldsymbol{\zeta}^{(1)} \\
\vdots \\
\boldsymbol{\zeta}^{(J)}
\end{array}\right]
$$

and a similar notation is used for $\mathbf{u}_{k}$ and $\mathbf{d}_{k}$.

Lines 4 and 6 of this algorithm are the main steps and those that can pose computational challenges. These steps, however, were shown to have fast closed-form solutions in several cases of interest $[1,13,18,21]$. In particular, the matrix inversion in line 4 can sometimes (e.g., in periodic deconvolution problems) be computed cheaply, by exploiting the matrix inversion lemma, the FFT and other fast transforms (see $[1,13]$ ), while line 6 corresponds to a so-called Moreau proximity operator (MPO), defined (for some function $f$ ) as

$$
\operatorname{prox}_{f}(\mathbf{y})=\arg \min _{\mathbf{x}} \frac{1}{2}\|\mathbf{y}-\mathbf{x}\|_{2}^{2}+f(\mathbf{x}) ;
$$

for several choices of $f, \operatorname{prox}_{f}$ has a simple closed form [7].

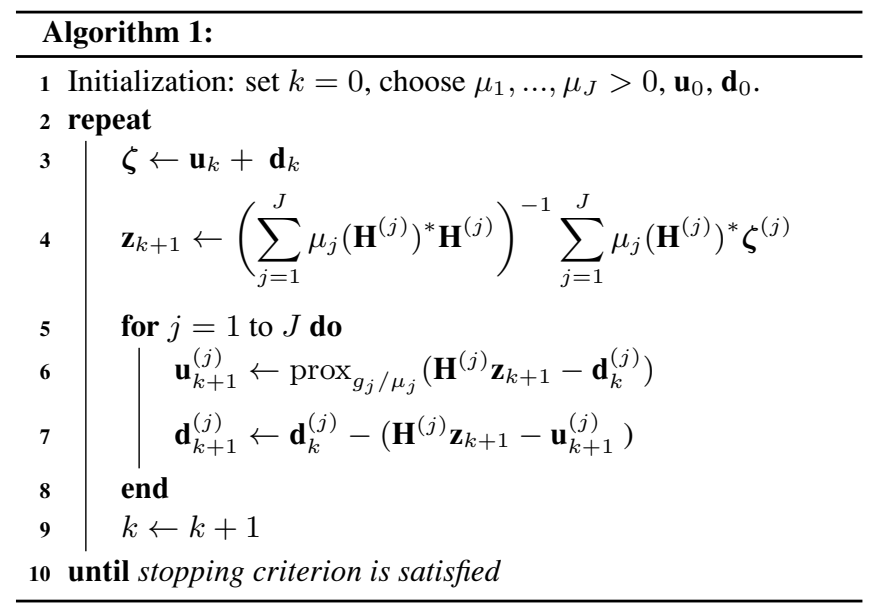

Under the condition that (1) has a solution, Algorithm 1 inherits the convergence guarantees of ADMM given in [11]. For our formulation, sufficient conditions for Algorithm 1 to converge to a solution of 1 are: $\mu_{1}, \ldots, \mu_{J}>0$; all functions $g_{j}$ are proper, closed, and convex; the matrix $\mathbf{G}=\left[\left(\mathbf{H}^{(1)}\right)^{*} \ldots\left(\mathbf{H}^{(J)}\right)^{*}\right]^{*} \in \mathbb{R}^{p \times d}$ has full column rank (where ()$^{*}$ denotes matrix/vector conjugate transpose, and $p=\sum_{j} p_{j}$.

\section{PROPOSED APPROACH}

This section introduces the proposed approach for deblurring with unknown boundaries. The description will use the frame-based analysis formulation, arguably one of the standard forms of regularization for this class of imaging inverse problems $[1,2,13,20]$. Extension to other popular regularizers, namely total variation (TV) or frame-based synthesis formulations is straightforward.

\subsection{Deconvolution with periodic BC}

We begin by considering the usual observation model used in image deconvolution with periodic BC: $\mathbf{y}=\mathbf{A x}+\mathbf{w}$, where $\mathbf{x} \in \mathbb{R}^{n}$ and $\mathbf{y} \in \mathbb{R}^{n}$ are vectors containing all pixels (lexicographically ordered) of the original and the observed images, respectively, w de- notes white Gaussian noise, and $\mathbf{A} \in \mathbb{R}^{n \times n}$ is the matrix representing the (periodic) convolution with some filter. In the frame-based analysis approach, the estimated image, $\widehat{\mathbf{x}} \in \mathbb{R}^{n}$, is obtained as

$$
\widehat{\mathbf{x}}=\arg \min _{\mathbf{x} \in \mathbb{R}^{n}} \frac{1}{2}\|\mathbf{y}-\mathbf{A x}\|_{2}^{2}+\lambda \phi(\mathbf{P x}),
$$

where $\mathbf{P} \in \mathbb{R}^{q \times n}(q \geq n)$ is the analysis operator of some frame (e.g., a redundant wavelet frame or a curvelet frame [15]), $\phi$ is a regularizer encouraging the vector of frame analysis coefficients to be sparse, and $\lambda>0$ is the regularization parameter. A typical choice for $\phi$, herein adopted, is $\phi(\mathbf{z})=\|\mathbf{z}\|_{1}=\sum_{i}\left|z_{i}\right|$.

Problem (2) can be written in the form (1), with $J=2$ and

$$
\begin{array}{ll}
g_{1}: \mathbb{R}^{n} \rightarrow \mathbb{R}, & g_{1}(\mathbf{v})=\frac{1}{2}\|\mathbf{y}-\mathbf{v}\|_{2}^{2}, \\
g_{2}: \mathbb{R}^{q} \rightarrow \mathbb{R}, & g_{2}(\mathbf{z})=\lambda\|\mathbf{z}\|_{1}, \\
\mathbf{H}^{(1)} \in \mathbb{R}^{n \times n}, & \mathbf{H}^{(1)}=\mathbf{A}, \\
\mathbf{H}^{(2)} \in \mathbb{R}^{q \times n}, & \mathbf{H}^{(2)}=\mathbf{P} .
\end{array}
$$

The Moreau proximity operators of $g_{1}$ and $g_{2}$, key components of Algorithm 1 (line 6), have simple expressions,

$$
\begin{aligned}
& \operatorname{prox}_{g_{1} / \mu_{1}}(\mathbf{v})=\frac{\mathbf{y}+\mu_{1} \mathbf{v}}{1+\mu_{1}}, \\
& \operatorname{prox}_{g_{2} / \mu_{2}}(\mathbf{z})=\operatorname{soft}\left(\mathbf{z}, \frac{\lambda}{\mu_{2}}\right),
\end{aligned}
$$

where "soft" denotes the well-known soft-threshold function

$$
\operatorname{soft}(\mathbf{v}, \gamma)=\operatorname{sign}(\mathbf{v}) \odot \max \{|\mathbf{v}|-\tau, 0\},
$$

where the sign, max, and absolute value functions are componentwise, and $\odot$ denotes the component-wise product.

Line 4 of Algorithm 1 (the other key component) has the form

$$
\mathbf{z}_{k+1} \leftarrow\left(\mathbf{A}^{*} \mathbf{A}+\frac{\mu_{2}}{\mu_{1}} \mathbf{P}^{*} \mathbf{P}\right)^{-1}\left(\mathbf{A}^{*} \boldsymbol{\zeta}^{(1)}+\frac{\mu_{2}}{\mu_{1}} \mathbf{P}^{*} \boldsymbol{\zeta}^{(2)}\right) .
$$

Assuming that $\mathbf{P}$ corresponds to a Parseval ${ }^{1}$ frame (i.e., $\mathbf{P}^{*} \mathbf{P}=\mathbf{I}$, although possibly $\mathbf{P} \mathbf{P}^{*} \neq \mathbf{I}$ ), the matrix inverse in (10) is simply computed in the DFT domain

$$
\left(\mathbf{A}^{*} \mathbf{A}+\frac{\mu_{2}}{\mu_{1}} \mathbf{I}\right)^{-1}=\mathbf{U}^{*}\left(|\boldsymbol{\Lambda}|^{2}+\frac{\mu_{2}}{\mu_{1}} \mathbf{I}\right)^{-1} \mathbf{U}
$$

in which $\mathbf{U}$ and $\mathbf{U}^{*}$ are the unitary matrices representing the DFT and its inverse, and $\Lambda$ is the diagonal matrix of the DFT coefficients of the convolution kernel (i.e., $\mathbf{A}=\mathbf{U}^{*} \boldsymbol{\Lambda} \mathbf{U}$ ).

The inversion in (11) has $O(n \log n)$ cost, since matrix $\left(|\boldsymbol{\Lambda}|^{2}+\right.$ $\left.\left(\mu_{2} / \mu_{1}\right) \mathbf{I}\right)$ is diagonal and the products by $\mathbf{U}$ and $\mathbf{U}^{*}$ (the DFT and its inverse) can be computed using the FFT. The leading cost of each application of (10) (line 4 of Algorithm 1) is thus either the $O(n \log n)$ cost associated with (11) or the cost of the products by $\mathbf{P}^{*}$. For most tight frames used in image restoration, this product has fast $O(n \log n)$ algorithms [15]. We conclude that, under periodic $\mathrm{BC}$ and for a large class of frames, each iteration of Algorithm 1 for solving (2) has $O(n \log n)$ cost.

Finally, this instance of ADMM has convergence guarantees, since: (1) $g_{2}$ is coercive, so is the objective function in (2), thus its set of minimizers is not empty [8]; (2) $g_{1}$ and $g_{2}$ are proper, closed,

\footnotetext{
${ }^{1}$ Obviously, we only need the frame to be tight $\left(\mathbf{P}^{*} \mathbf{P}=\omega \mathbf{I},[15]\right)$, but assuming $\omega=1$ lightens the notation and doesn't cause loss of generality.
} 
convex functions; (3) matrix $\mathbf{H}^{(2)}=\mathbf{I}$ obviously has full column rank, which implies that $\mathbf{G}=\left[\mathbf{A}^{*} \mathbf{I}^{*}\right]^{*}$ also has full column rank.

\subsection{Deconvolution with unknown boundaries}

In the unknown boundary model, the observed image $\mathbf{y} \in \mathbb{R}^{m}$ depends on a larger sharp image $\mathbf{x} \in \mathbb{R}^{n}$ that contains unobserved boundary pixels,

$$
\mathbf{y}=\mathbf{M A} \mathbf{x}+\mathbf{n},
$$

where $\mathbf{M} \in\{0,1\}^{m \times n}$ (with $m<n$ ) is a masking matrix, whose role is to observe only the subset of the image domain in which the elements of $\mathbf{A x}$ do not depend on the boundary pixels. For a blurring filter of a limited support of size $(1+2 l) \times(1+2 l)$, matrix $\mathbf{M}$ removes a band of width $l$ of the outermost pixels of the full convolved image $\mathbf{A x}$. The BC assumed for the convolution represented by $\mathbf{A}$ is thus irrelevant, and we may adopt periodic BCs, for computational convenience.

Problem (12) can be seen as hybrid of deconvolution and inpainting [6], where the missing pixels constitute the unknown boundary. Inpainting and periodic deconvolution are thus particular cases of this general model (obtained for $\mathbf{A}=\mathbf{I}$ and $\mathbf{M}=\mathbf{I}$, respectively). This model is also suitable for problems where some interior pixels are missing; e.g., in a deblurring problem with saturated (thus unreliable) pixels, or in a super-resolution problem. More generally, matrix $\mathbf{M}$ can take values in $[0,1]$, allowing to differently weight the observed pixels; this case allows spatially varying regularization, but will not be considered in this paper.

Under model (12), the frame-based analysis formulation (2) changes to

$$
\widehat{\mathbf{x}}=\arg \min _{\mathbf{x} \in \mathbb{R}^{m}} \frac{1}{2}\|\mathbf{y}-\mathbf{M A x}\|_{2}^{2}+\lambda\|\mathbf{P} \mathbf{x}\|_{1} .
$$

At this point, one could be tempted to map (13) into (1) using (3), (4), and (6), and simply change (5) into

$$
\mathbf{H}^{(1)} \in \mathbb{R}^{m \times n}, \quad \mathbf{H}^{(1)}=\mathbf{M A} .
$$

The problem with this choice is that the matrix to be inverted in line 4 of Algorithm 1 would become

$$
\left(\mathbf{A}^{*} \mathbf{M}^{*} \mathbf{M A}+\left(\mu_{2} / \mu_{1}\right) \mathbf{I}\right)
$$

which, unlike (10), is not easily invertible due to the presence of $\mathbf{M}$. To sidestep this difficulty, we propose to decouple the action of the (DFT diagonal) operator $\mathbf{A}$ from the spatial operator $\mathbf{M}$, by keeping (4), (5), and (6), and replacing (3) by

$$
g_{1}: \mathbb{R}^{n} \rightarrow \mathbb{R}, \quad g_{1}(\mathbf{v})=\frac{1}{2}\|\mathbf{y}-\mathbf{M v}\|_{2}^{2} .
$$

With this choice, line 4 of Algorithm 1 is still given by (10) (with its efficient FFT-based implementation (11)), while mask operator $\mathbf{M}$ only affects the Moreau proximity operator of the new $g_{1}$,

$$
\begin{aligned}
\operatorname{prox}_{g_{1} / \mu_{1}}(\mathbf{v}) & =\arg \min _{\mathbf{u}}\|\mathbf{M u}-\mathbf{y}\|_{2}^{2}+\mu_{1}\|\mathbf{u}-\mathbf{v}\|_{2}^{2} \\
& =\left(\mathbf{M}^{*} \mathbf{M}+\mu_{1} \mathbf{I}\right)^{-1}\left(\mathbf{M}^{*} \mathbf{y}+\mu_{1} \mathbf{v}\right) .
\end{aligned}
$$

Notice that, due to the special structure of $\mathbf{M}$, matrix $\mathbf{M}^{*} \mathbf{M}$ is diagonal, thus the inversion in (18) has $O(n)$ cost, the same being true about the product $\mathbf{M}^{*} \mathbf{y}$, which corresponds to extending the observed image $\mathbf{y}$ to the size of $\mathbf{x}$, by creating a boundary of zeros around it. Of course, both $\left(\mathbf{M}^{*} \mathbf{M}+\mu_{1} \mathbf{I}\right)^{-1}$ and $\mathbf{M}^{*} \mathbf{y}$ can be pre- computed and then used throughout the algorithm, as long as $\mu_{1}$ is kept constant.

Similarly to what was shown for the periodic BC case, the proposed ADMM approach for deblurring with unknown boundaries has a leading cost of $O(n \log n)$ per iteration. Considering the similarities of our approach with the one of Section 3.1, it is sufficient to confirm that the new $g_{1}$ in (16) is proper, closed, and convex (which is obviously the case), to guarantee the convergence of the proposed ADMM algorithm.

\section{EXPERIMENTS}

To test our approach, the $256 \times 256$ Lena image was degraded with four $19 \times 19$ blurs, namely, uniform (square), out-of-focus (circular), linear motion (at $45^{\circ}$ ), and Gaussian, followed by the addition of Gaussian white noise at $40 \mathrm{~dB}, 50 \mathrm{~dB}$, and $60 \mathrm{~dB}$ blurred signal to noise ratio (BSNR). The observed images contain only the region where the convolution does not depend on the boundaries: for $19 \times$ 19 blurs, the observed images contain $238 \times 238$ pixels.

On each degraded image, the algorithm proposed in Section 3.2 was run, as well as the periodic version (Section 3.1), with and without pre-processing the observed image with the "edgetapper" MATLAB function. The algorithms are stopped when $\left\|\mathbf{z}_{k}-\mathbf{z}_{k-1}\right\|_{2} /\left\|\mathbf{z}_{k}\right\|_{2}<10^{-3}$ and $\lambda$ was adjusted to yield the highest SNR of the reconstructed image.

Table 1 shows, for each blur and BSNR, the ISNR (improvement in SNR) values obtained with the three algorithms mentioned in the previous paragraph. The huge impact of wrongly assuming periodic BC is clear in these results, as well as in the example shown in Fig. 2. Although the "edgetaper" function is able to somewhat mitigate this effect, the visual results are far from acceptable. Notice also that (as can be seen in Fig. 2), the proposed approach produced a remarkably accurate estimate of the unobserved boundary region. Regarding computational cost, the algorithm for unknown boundaries is essentially similar to its periodic BC counterpart, which is known to achieve state-of-the-art convergence speed [1,2].

\begin{tabular}{cccc} 
& \multicolumn{3}{c}{ deconvolution method } \\
\cline { 2 - 4 } blur, BSNR & periodic & "edgetaper" & proposed \\
\hline uniform, 60dB & -2.52 & 3.06 & 10.63 \\
\hline out-of-focus, 60dB & -1.50 & 5.04 & 14.21 \\
\hline linear motion, 60dB & -1.84 & 7.31 & 16.41 \\
\hline Gaussian, 60dB & -0.33 & 3.94 & 4.69 \\
\hline \hline uniform, 50dB & -2.53 & 3.06 & 9.02 \\
\hline out-of-focus, 50dB & -1.50 & 5.02 & 10.99 \\
\hline linear motion, 50dB & -1.84 & 7.29 & 14.63 \\
\hline Gaussian, 50dB & -0.33 & 3.87 & 4.21 \\
\hline \hline uniform, 40dB & -2.54 & 3.05 & 6.83 \\
\hline out-of-focus, 40dB & -1.50 & 4.88 & 7.95 \\
\hline linear motion, 40dB & -1.84 & 7.12 & 11.22 \\
\hline Gaussian, 40dB & -0.33 & 3.48 & 3.48 \\
\hline \hline average & $\mathbf{- 1 . 4 0}$ & $\mathbf{4 . 7 6}$ & $\mathbf{9 . 5 2}$ \\
\hline
\end{tabular}

Table 1. ISNR values achieved by the 3 tested approaches (see text).

\section{CONCLUSIONS AND FUTURE WORK}

We proposed a new strategy to extend fast ADMM-based algorithms in order to address deconvolution problems with unknown bound- 


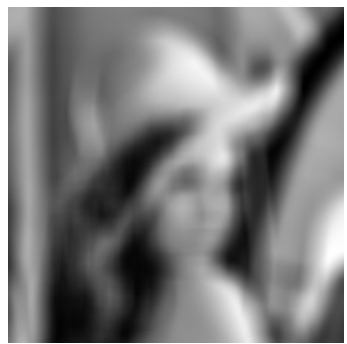

observed $(238 \times 238)$

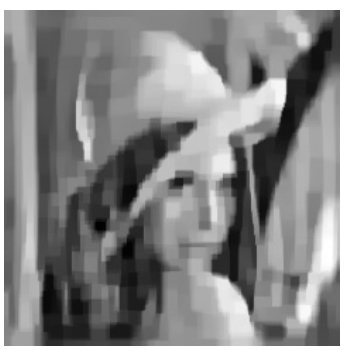

edgetaper $(\mathrm{ISNR}=3.06 \mathrm{~dB})$
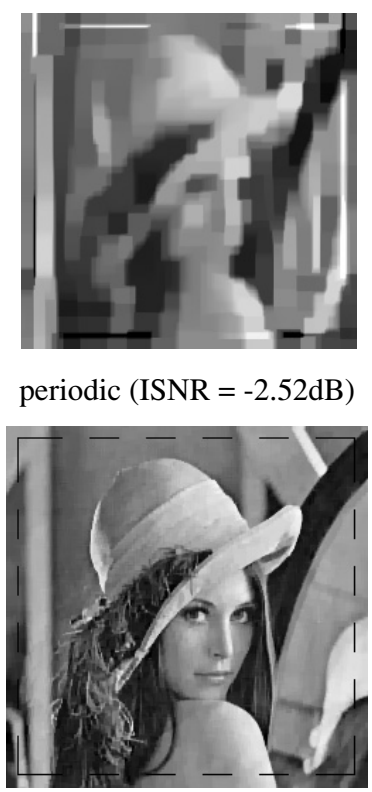

proposed $(\mathrm{ISRN}=10.63 \mathrm{~dB})$ periodic $(\mathrm{ISNR}=-2.52 \mathrm{~dB})$

Fig. 2. Results obtained on the Lena image, degraded by a uniform $19 \times 19$ blur at $60 \mathrm{~dB}$ BSNR, by the three algorithms considered (see text). Notice that the algorithms that assume periodic BC (in addition to huge artifacts) produce $238 \times 238$ images, while the proposed algorithm yields $256 \times 256$ images, by estimating the unknown boundary (marked by the dashed square).

aries, while previous versions were limited to periodic boundary conditions. The proposed approach was illustrated using a framebased analysis formulation, and the resulting algorithm was shown to inherit the convergence guarantees of ADMM and to have the same computational cost per iteration as the version that assumes periodic boundary conditions.

Besides allowing deblurring with unknown boundaries, the proposed ADMM approach is also applicable to a more general class of inverse problems, such as super-resolution and simultaneous inpainting and deblurring; these will be topics of future work.

\section{REFERENCES}

[1] M. Afonso, J. Bioucas-Dias, and M. Figueiredo, "Fast image recovery using variable splitting and constrained optimization," IEEE Trans. Image Proc., vol. 19, pp. 2345-2356, 2010.

[2] _ - "An augmented Lagrangian approach to the constrained optimization formulation of imaging inverse problems," IEEE Trans. Image Proc., vol. 20, pp. 681-695, 2011.

[3] A. Beck and M. Teboulle, "A fast iterative shrinkagethresholding algorithm for linear inverse problems," SIAM Jour. Imaging Sciences, vol. 2, pp. 183-202, 2009.

[4] J. Bioucas-Dias and M. Figueiredo, "A new TwIST: two-step iterative shrinkage/thresholding algorithms for image restoration," IEEE Trans. Image Proc., vol. 16, pp. 2992-3004, 2007.

[5] S. Boyd, N. Parikh, E. Chu, B. Peleato, and J. Eckstein, "Distributed optimization and statistical learning via the alternating direction method of multipliers," Foundations and Trends in Machine Learning, vol. 3, pp. 1-122, 2011.

[6] T. Chan, A. Yip, and F. Park, "Simultaneous total variation image inpainting and blind deconvolution," International Journal of Imaging Systems and Technology, vol. 15, pp. 92-102, 2005.

[7] P. Combettes and J.-C. Pesquet, "Proximal Splitting Methods in Signal Processing", in Fixed-Point Algorithms for Inverse Problems in Science and Engineering (H. Bauschke et al, Editors), pp. 185-212, Springer, 2011.

[8] P. Combettes and V. Wajs, "Signal recovery by proximal forward-backward splitting," SIAM Journal on Multiscale Modeling \& Simulation, vol. 4, pp. 1168-1200, 2005.

[9] I. Daubechies, M. Defrise, C. De Mol, "An iterative thresholding algorithm for linear inverse problems with a sparsity constraint," Comm. Pure and App. Math., vol. 57, pp. 1413-1457, 2004.

[10] M. Donatelli, C. Estatico, A. Martinelli, and S. SerraCapizzano, "Improved image deblurring with anti-reflective boundary conditions and re-blurring," Inverse Problems, vol. 22, pp. 2035-2053, 2006.

[11] J. Eckstein and D. Bertsekas, "On the Douglas-Rachford splitting method and the proximal point algorithm for maximal monotone operators," Math. Prog., vol. 55, pp. 293-318, 1992.

[12] M. Figueiredo and R. Nowak, "An EM algorithm for waveletbased image restoration," IEEE Trans. Image Proc., vol. 12, pp. 906-916, 2003.

[13] M. Figueiredo and J. Bioucas-Dias, "Restoration of Poissonian images using alternating direction optimization," IEEE Trans. Image Proc., vol. 19, pp. 3133-314, 2010.

[14] D. Gabay and B. Mercier, "A dual algorithm for the solution of nonlinear variational problems via infinite element approximations," Comput. and Math. with Appl., vol. 2, p. 17-40, 1976.

[15] S. Mallat, A Wavelet Tour of Signal Processing. Academic Press, 2009.

[16] A. Matakos, S. Ramani, and J. Fessler, "Image restoration using non-circulant shift-invariant system models." Proc. IEEE Intl. Conf. Image Proc., pp. 3061-3064, 2012.

[17] M. Ng, Iterative methods for Toeplitz systems. Oxford University Press, 2004.

[18] S. Ramani and J. Fessler, "A splitting-based iterative algorithm for accelerated statistical X-ray CT reconstruction," IEEE Trans. Medical Imag., vol. 31, pp. 677-688, 2012.

[19] S. Reeves, "Fast image restoration without boundary artifacts," IEEE Trans. Image Proc., vol. 14, pp. 1448-1453, 2005.

[20] I. Selesnick and M. Figueiredo, "Signal restoration with overcomplete wavelet transforms: Comparison of analysis and synthesis priors," in Proceedings of SPIE, vol. 7446, 2009.

[21] G. Steidl and T. Teuber, "Removing multiplicative noise by Douglas-Rachford splitting methods," Journal of Mathematical Imaging and Vision, vol. 36, pp. 168-184, 2010.

[22] M. Šorel, "Removing boundary artifacts for real-time iterated shrinkage deconvolution," IEEE Trans. Image Proc., vol. 21, pp. 2329-2334, 2012.

[23] S. Wright, R. Nowak, and M. Figueiredo, "Sparse reconstruction by separable approximation," IEEE Trans. Signal Proc., vol. 57, pp. 2479-2493, 2009. 